\title{
Resonant $p$-Laplacian problems with functional boundary conditions
}

\section{Weihua Jiang ${ }^{1 *}$ (D) and Nickolai Kosmatov ${ }^{2}$}

\section{"Correspondence:}

jianghua64@163.com

${ }^{1}$ College of Sciences, Hebei

University of Science and

Technology, Shijiazhuang, P.R. China

Full list of author information is

available at the end of the article

\section{Introduction}

A boundary value problem is said to be at resonance if the corresponding homogeneous boundary value problem has a non-trivial solution. Mawhin's continuation theorem [1] and its extension by Ge and Ren [2] are effective tools in the study of boundary value problems at resonance (see [3-10] and the references therein). In Refs. [6, 9], the authors studied the existence of solutions for functional boundary value problems with a linear differential operator by using Mawhin's continuation theorem. In [6], we extended the results of [9] to include new resonance scenarios. Since the $p$-Laplacian operator occurs in many applications such as non-Newtonian mechanics, nonlinear elasticity and glaciology, combustion theory, we would like to further extend the results of [6] to the third-order functional $p$-Laplacian boundary value problem at resonance

$$
\left\{\begin{array}{l}
\left(\varphi_{p}\left(u^{\prime \prime}\right)\right)^{\prime}(t)=f\left(t, u(t), u^{\prime}(t), u^{\prime \prime}(t)\right), \quad t \in(0,1), \\
u^{\prime \prime}(0)=0, \quad B_{1}(u)=B_{2}(u)=0,
\end{array}\right.
$$

where $f:[0,1] \times \mathbb{R}^{3} \rightarrow \mathbb{R}$ is continuous, $p>1, \varphi_{p}(s)=|s|^{p-2} s, B_{1}, B_{2}: C^{2}[0,1] \rightarrow \mathbb{R}$ are linear bounded functions with $B_{2}(t) B_{1}(1)=B_{2}(1) B_{1}(t)$. Although the paper by Han and Kang [11] explores positive solutions in the non-resonant setting of a dynamic equation on a measure chain with Sturm-Liouville boundary conditions in place of our functional conditions, it is also relevant since it bears some similarity to the boundary value problem considered herein. Finally, one can easily extend the scheme used in this paper to include fractional analogs of [4] and thus to extend the findings of [10].

(c) The Author(s) 2018. This article is distributed under the terms of the Creative Commons Attribution 4.0 International License (http://creativecommons.org/licenses/by/4.0/), which permits unrestricted use, distribution, and reproduction in any medium, provided you give appropriate credit to the original author(s) and the source, provide a link to the Creative Commons license, and indicate if changes were made. 


\section{Preliminaries}

We introduce the theoretical foundations of the method; for more details, see [2].

Definition 2.1 Let $X$ and $Y$ be two Banach spaces with norms $\|\cdot\|_{X},\|\cdot\|_{Y}$, respectively. An operator $M: X \cap \operatorname{dom} M \rightarrow Y$ is said to be quasi-linear if

(i) $\operatorname{Im} M=M(X \cap \operatorname{dom} M)$ is a closed subset of $Y$;

(ii) $\operatorname{Ker} M=\{x \in X \cap \operatorname{dom} M: M x=0\}$ is linearly homeomorphic to $\mathbb{R}^{n}$.

In this paper, an operator $T: X \rightarrow Y$ is said to be bounded if $T(V) \subset Y$ is bounded for any bounded subset $V \subset X$.

Definition 2.2 A linear operator $P: X \rightarrow X$, where $X$ is a vector space, is a projector if $P^{2} x=P x$.

Let $X_{1}=\operatorname{Ker} M, P: X \rightarrow X_{1}$ be a projector and $X_{2}$ be the complement space of $X_{1}$ in $X$ with $X=X_{1} \oplus X_{2}$. Let $\Omega \subset X$ be an open and bounded set with the origin $0 \in \Omega$.

Definition 2.3 Suppose that $N_{\lambda}: \bar{\Omega} \rightarrow Y, \lambda \in[0,1]$ is a continuous and bounded operator and $N_{1}$ is denoted by $N$. Let $\Sigma_{\lambda}=\left\{x \in \bar{\Omega}: M x=N_{\lambda} x\right\}$. The operator $N_{\lambda}$ is said to be $M$ quasi-compact in $\bar{\Omega}$ if there exists a vector subspace $Y_{1}$ of $Y$ satisfying $\operatorname{dim} Y_{1}=\operatorname{dim} X_{1}$ and the operators $Q$ and $R$ such that the following conditions hold:

(a) $\operatorname{Ker} Q=\operatorname{Im} M$;

(b) $Q N_{\lambda} x=0, \lambda \in(0,1) \Leftrightarrow Q N x=0$;

(c) $R(\cdot, 0)$ is the zero operator and $\left.R(\cdot, \lambda)\right|_{\Sigma_{\lambda}}=\left.(I-P)\right|_{\Sigma_{\lambda}}$;

(d) $M[P+R(\cdot, \lambda)]=(I-Q) N_{\lambda}$, where $Q: Y \rightarrow Y_{1}$ is continuous, bounded with $Q(I-Q)=0, Q Y=Y_{1}$ and $R: \bar{\Omega} \times[0,1] \rightarrow X_{2}$ is continuous and compact with $P u+R(u, \lambda) \in \operatorname{dom} M, u \in \bar{\Omega}, \lambda \in[0,1]$.

We use the result of Ge and Ren [2].

Theorem 2.4 Let $X$ and $Y$ be Banach spaces and $\Omega \subset X$ be an open and bounded nonempty set. Suppose that $M: X \cap \operatorname{dom} M \rightarrow Y$ is a quasi-linear operator and $N_{\lambda}: \bar{\Omega} \rightarrow Y$, $\lambda \in[0,1]$, is $M$-quasi-compact. In addition, if the following conditions hold:

$\left(C_{1}\right) M x \neq N_{\lambda} x, x \in \partial \Omega \cap \operatorname{dom} M, \lambda \in(0,1)$;

$\left(C_{2}\right) \operatorname{deg}(J Q N, \Omega \cap \operatorname{Ker} M, 0) \neq 0$, where $N=N_{1}, J: \operatorname{Im} Q \rightarrow \operatorname{Ker} M$ is a homeomorphism with $J(0)=0$ and deg is the Brouwer degree,

then the abstract equation $M x=N x$ has at least one solution in $\operatorname{dom} M \cap \bar{\Omega}$.

We make use of well-known inequalities [12] in the context of the $p$-Laplacian $\varphi_{p}(s)$, $p>1$. For $u, v \geq 0$, we have

$$
\varphi_{p}(u+v) \leq \begin{cases}\varphi_{p}(u)+\varphi_{p}(v), & \text { if } 1<p \leq 2, \\ 2^{p-2}\left(\varphi_{p}(u)+\varphi_{p}(v)\right), & \text { if } p>2 .\end{cases}
$$

\section{Main results}

We work in the Banach spaces $X=\left\{u \in C^{2}[0,1]: u^{\prime \prime}(0)=0\right\}$ with the norm $\|u\|_{X}=$ $\max \left\{\|u\|_{0},\left\|u^{\prime}\right\|_{0},\left\|u^{\prime \prime}\right\|_{0}\right\}$ and $Y=C[0,1]$ with the norm $\|y\|_{Y}=\|y\|_{0}$, where $\|\cdot\|_{0}$ is the max-norm and introduce the following assumptions: 
$\left(A_{0}\right)$ The linear functionals $B_{i}: X \rightarrow \mathbb{R}, i=1,2$, satisfy $B_{1}(t)=\beta, B_{1}(1)=\alpha, B_{2}(t)=k \beta$, $B_{2}(1)=k \alpha$, where $\alpha, \beta, k \in \mathbb{R}$ with $\alpha^{2}+\beta^{2} \neq 0$.

$\left(A_{1}\right)\left\|B_{i} u\right\| \leq k_{i}\|u\|_{X}, k_{i} \in \mathbb{R}^{+}, u \in X, i=1,2$.

$\left(A_{2}\right)$ The functional $F: Y \rightarrow \mathbb{R}$ defined by

$$
F(y)=\left(B_{2}-k B_{1}\right)\left(\int_{0}^{t}(t-s) \varphi_{q}\left(\int_{0}^{s} y(r) d r\right) d s\right)
$$

where $\frac{1}{p}+\frac{1}{q}=1$ is increasing, that is, if $y_{1}, y_{2} \in Y, y_{1}(t) \leq y_{2}(t), t \in[0,1], y_{1} \not \equiv y_{2}$, then $F\left(y_{1}\right)<F\left(y_{2}\right)$.

Define operators $M: X \cap \operatorname{dom} M \rightarrow Y$ and $N_{\lambda}: X \rightarrow Y$ by

$$
M u(t)=\left(\varphi_{p}\left(u^{\prime \prime}\right)\right)^{\prime}(t)
$$

where $\operatorname{dom} M=\left\{u \in X: B_{1}(u)=B_{2}(u)=0,\left(\varphi_{p}\left(u^{\prime \prime}\right)\right)^{\prime} \in C[0,1]\right\}$, and

$$
N_{\lambda} u(t)=\lambda f\left(t, u(t), u^{\prime}(t), u^{\prime \prime}(t)\right), \quad \lambda \in[0,1] .
$$

It is easy to see recalling (3.1) that

$$
\operatorname{Ker} M=\{c(\alpha t-\beta): c \in \mathbb{R}\} \quad \text { and } \quad \operatorname{Im} M=\{y \in Y: F(y)=0\} .
$$

In fact, if $y \in \operatorname{Im} M$, there exists a function $u \in \operatorname{dom} M$ with $M u=y$. So,

$$
u(t)=\int_{0}^{t}(t-s) \varphi_{q}\left(\int_{0}^{s} y(r) d r\right) d s+a t+b, \quad a, b \in \mathbb{R}
$$

By $B_{i}(u)=0$, we get

$$
\begin{aligned}
& B_{1}(u)=B_{1}\left(\int_{0}^{t}(t-s) \varphi_{q}\left(\int_{0}^{s} y(r) d r\right) d s\right)+a \beta+b \alpha=0, \\
& B_{2}(u)=B_{2}\left(\int_{0}^{t}(t-s) \varphi_{q}\left(\int_{0}^{s} y(r) d r\right) d s\right)+a k \beta+b k \alpha=0 .
\end{aligned}
$$

Thus, $F(y)=0$.

Conversely, if $y \in Y$ satisfies $F(y)=0$, we let

$$
\begin{aligned}
u(t)= & \int_{0}^{t}(t-s) \varphi_{q}\left(\int_{0}^{s} y(r) d r\right) d s \\
& -\frac{1}{\alpha^{2}+\beta^{2}} B_{1}\left(\int_{0}^{t}(t-s) \varphi_{q}\left(\int_{0}^{s} y(r) d r\right) d s\right)(\beta t+\alpha) .
\end{aligned}
$$

Clearly, $u^{\prime \prime}(0)=0,\left(\varphi_{p}\left(u^{\prime \prime}\right)\right)^{\prime}=y$ and $B_{1}(u)=B_{2}(u)=0$. Therefore, $u \in \operatorname{dom} M$ and $M u=y$, that is, $y \in \operatorname{Im} M$.

Obviously, $\operatorname{Ker} M$ is linearly homeomorphic to $\mathbb{R}$. Let $y_{n} \in \operatorname{Im} M \subset Y, y_{n} \rightarrow y \in Y$. Since

$$
\left\|\int_{0}^{t}(t-s) \varphi_{q}\left(\int_{0}^{s} y_{n}(r) d r\right) d s-\int_{0}^{t}(t-s) \varphi_{q}\left(\int_{0}^{s} y(r) d r\right) d s\right\|_{X} \rightarrow 0, \quad \text { as } n \rightarrow \infty,
$$


then, by $\left(A_{1}\right),\left|F\left(y_{n}\right)-F(y)\right| \rightarrow 0$ as $n \rightarrow \infty$. This, together with $y_{n} \in \operatorname{Im} M$, shows that $y \in \operatorname{Im} M$. Hence, $\operatorname{Im} M$ is a closed subset of $Y$. Thus, $M$ is quasi-linear. Set $X_{1}=\operatorname{Ker} M$. Define operators $P: X \rightarrow X$ and $Q: Y \rightarrow Y$ by

$$
P u=\frac{\alpha u^{\prime}(0)-\beta u(0)}{\alpha^{2}+\beta^{2}}(\alpha t-\beta),
$$

and $Q y=c$, where $c$ satisfies $F(y-c)=0$.

Clearly, $P$ is a projector and $\operatorname{Ker} Q=\operatorname{Im} M$. Set $Y_{1}=\mathbb{R}$.

Lemma 3.1 The operator $Q: Y \rightarrow Y_{1}$ is continuous, bounded and $Q(I-Q)=0, Q Y=Y_{1}$, $|Q y| \leq\|y\|_{Y}$.

Proof For $y \in Y$, by $\left(A_{1}\right)$ and $\left(A_{2}\right)$, it follows that the function $F(y-\cdot): \mathbb{R} \rightarrow \mathbb{R}$, defined in terms of (3.1), is continuous and decreasing. Choose $a, b \in \mathbb{R}$ and $y \in Y$ such that $a>$ $\|y\|_{Y}, b<-\|y\|_{Y}$. By $\left(A_{2}\right), F(y-a)<0<F(y-b)$. So, there exists a unique constant $c$ with $|c| \leq\|y\|_{Y}$ such that $F(y-c)=0$. Thus, $Q$ is well defined and $|Q y| \leq\|y\|_{Y}$. For $y_{1}, y_{2} \in Y$, $Q\left(y_{1}\right)=c_{1}, Q\left(y_{2}\right)=c_{2}$, if $c_{2}-c_{1}>\left\|y_{2}-y_{1}\right\|_{Y}$, it follows from $\left(A_{2}\right)$ that

$$
0=F\left(y_{1}-c_{1}\right)=F\left(y_{2}-c_{2}-\left[\left(y_{2}-y_{1}\right)-\left(c_{2}-c_{1}\right)\right]\right)>F\left(y_{2}-c_{2}\right)=0,
$$

which is a contradiction. If $c_{2}-c_{1}<-\left\|y_{2}-y_{1}\right\|_{Y}$, then

$$
0=F\left(y_{1}-c_{1}\right)=F\left(y_{2}(r)-c_{2}-\left[\left(y_{2}-y_{1}\right)-\left(c_{2}-c_{1}\right)\right]\right)<F\left(y_{2}-c_{2}\right)=0,
$$

which is a contradiction, again. Thus, $\left|Q\left(y_{2}\right)-Q\left(y_{1}\right)\right|=\left|c_{2}-c_{1}\right| \leq\left\|y_{2}-y_{1}\right\|_{Y}$, that is, $Q$ is continuous.

Obviously, $Q(I-Q)=0$ and $Q Y=Y_{1}$.

We define $R(u, \lambda): X \times[0,1] \rightarrow X_{2}$ by

$$
\begin{aligned}
R(u, \lambda)(t)= & \int_{0}^{t}(t-s) \varphi_{q}\left(\int_{0}^{s}(I-Q) N_{\lambda} u(r) d r\right) d s \\
& -\frac{1}{\alpha^{2}+\beta^{2}} B_{1}\left(\int_{0}^{t}(t-s) \varphi_{q}\left(\int_{0}^{s}(I-Q) N_{\lambda} u(r) d r\right) d s\right)(\beta t+\alpha),
\end{aligned}
$$

where $X_{1} \oplus X_{2}=X$.

Lemma 3.2 The operator $R: \bar{\Omega} \times[0,1] \rightarrow X_{2}$ is continuous and compact with Pu + $R(u, \lambda) \in \operatorname{dom} M, u \in \bar{\Omega}, \lambda \in[0,1]$, where $\Omega \subset X$ is bounded.

Proof Since $P R(u, \lambda)=0, R(u, \lambda) \in X_{2}$. For $u \in X, \lambda \in[0,1]$, it follows from the continuity of $B_{1}, Q$ and $f$ that $R(u, \lambda)$ is continuous. Clearly, $\left(\varphi_{p}\left((P u+R(u, \lambda))^{\prime \prime}\right)\right)^{\prime}=(I-Q) N_{\lambda} u \in C[0,1]$, $(P u+R(u, \lambda))^{\prime \prime}(0)=0$ and $B_{1}(P u+R(u, \lambda))=0$. Considering $(I-Q) N_{\lambda} u \in \operatorname{Ker} Q=\operatorname{Im} M$, we get $B_{2}(P u+R(u, \lambda))=0$. So, $P u+R(u, \lambda) \in \operatorname{dom} M$. Now, we prove that $R$ is compact.

There exists a constant $C>0$ such that $\left\|N_{\lambda} u\right\|_{Y} \leq C$ in $\bar{\Omega}$ for all $\lambda \in[0,1]$. Note that

$$
\left|(R(u, \lambda))^{\prime \prime}(t)\right|=\left|\varphi_{q}\left(\int_{0}^{t}(I-Q) N_{\lambda} u(s) d s\right)\right| \leq(2 C)^{q-1},
$$


$(R(u, \lambda))^{\prime \prime}$ is uniformly bounded in $\bar{\Omega}$ together with $R(u, \lambda)$ and $(R(u, \lambda))^{\prime}$. Also, since $\varphi_{q}(\cdot)$ is uniformly continuous in $[-2 C, 2 C]$ and, for $0 \leq t_{1}<t_{2} \leq 1$,

$$
\left|\int_{0}^{t_{2}}(I-Q) N_{\lambda} u(s) d s-\int_{0}^{t_{1}}(I-Q) N_{\lambda} u(s) d s\right| \leq 2 C\left(t_{2}-t_{1}\right)
$$

it follows that $\left.\{R(u, \lambda))^{\prime \prime}: u \in \bar{\Omega}, \lambda \in[0,1]\right\}$ is equicontinuous. By the mean value theorem, $\left\{(R(u, \lambda))^{\prime}: u \in \bar{\Omega}, \lambda \in[0,1]\right\}$ and $\{R(u, \lambda): u \in \bar{\Omega}, \lambda \in[0,1]\}$ are also equicontinuous. The compactness of the operator $R$ follows from the Arzela-Ascoli theorem.

Now, we will show that $N_{\lambda}$ is M-quasi-compact in $\bar{\Omega}$, where $\Omega \subset X$ is an open and bounded set with $0 \in \Omega$.

Obviously, $N_{\lambda}$ is continuous, bounded and $\operatorname{dim} X_{1}=\operatorname{dim} Y_{1}$.

Lemma 3.3 The operator $N_{\lambda}$ is M-quasi-compact in $\bar{\Omega}$.

Proof Obviously, $\operatorname{Ker} Q=\operatorname{Im} M, Q N_{\lambda} u=0, \lambda \in(0,1) \Leftrightarrow Q N u=0, R(\cdot, 0)$ is the zero operator and $M(P u+R(u, \lambda))=(I-Q) N_{\lambda} u$. Considering Lemmas 3.1 and 3.2, we need only to prove that $\left.R(\cdot, \lambda)\right|_{\sum_{\lambda}}=\left.(I-P)\right|_{\sum_{\lambda}}$.

To this end, $u \in \sum_{\lambda}$ implies $N_{\lambda} u=M u, u^{\prime \prime}(0)=0, B_{i}(u)=0, i=1,2$. Thus, $Q N_{\lambda} u=0$ and

$$
\begin{aligned}
R(u, \lambda)= & \int_{0}^{t}(t-s) \varphi_{q}\left(\int_{0}^{s} N_{\lambda} u(r) d r\right) d s \\
& -\frac{B_{1}\left(\int_{0}^{t}(t-s) \varphi_{q}\left(\int_{0}^{s} N_{\lambda} u(r) d r\right) d s\right)}{\alpha^{2}+\beta^{2}}(\beta t+\alpha) \\
= & \int_{0}^{t}(t-s) \varphi_{q}\left(\int_{0}^{s}\left(\varphi_{p}\left(u^{\prime \prime}\right)\right)^{\prime}(r) d r\right) d s \\
& -\frac{B_{1}\left(\int_{0}^{t}(t-s) \varphi_{q}\left(\int_{0}^{s}\left(\varphi_{p}\left(u^{\prime \prime}\right)\right)^{\prime}(r) d r\right) d s\right)}{\alpha^{2}+\beta^{2}}(\beta t+\alpha) \\
= & \int_{0}^{t}(t-s) u^{\prime \prime}(s) d s-\frac{B_{1}\left(\int_{0}^{t}(t-s) u^{\prime \prime}(s) d s\right)}{\alpha^{2}+\beta^{2}}(\beta t+\alpha) \\
= & u(t)-u^{\prime}(0) t-u(0)-\frac{-u^{\prime}(0) \beta-u(0) \alpha}{\alpha^{2}+\beta^{2}}(\beta t+\alpha) \\
= & u(t)-\frac{\alpha u^{\prime}(0)-\beta u(0)}{\alpha^{2}+\beta^{2}}(\alpha t-\beta) \\
= & (I-P) u .
\end{aligned}
$$

The proof is completed.

In order to obtain our main results, we need the following hypotheses:

$\left(H_{1}\right)$ There exists a constant $M_{0}>0$ such that if $|u(t)|+\left|u^{\prime}(t)\right|>M_{0}$, then $F(N u) \neq 0$.

$\left(H_{2}\right)$ There exist functions $a, b, c, d \in C[0,1]$ with $\|b\|_{1}+\|c\|_{1}+\|d\|_{1}<1$, if $1<p \leq 2$ and $2^{p-2}\left(\|b\|_{1}+\|c\|_{1}\right)+\|d\|_{1}<1$, if $p>2$, such that

$$
|f(t, u, v, w)| \leq a(t)+b(t) \varphi_{p}(|u|)+c(t) \varphi_{p}(|v|)+d(t) \varphi_{p}(|w|), \quad t \in[0,1], u, v, w \in \mathbb{R},
$$

where $\|y\|_{1}=\int_{0}^{1}|y(t)| d t$. 
$\left(H_{3}\right)$ There exists a constant $M_{1}>0$ such that for $|c|>M_{1}$ one of the following inequalities holds:

$$
\begin{aligned}
& c Q N(c(\alpha t-\beta))>0, \\
& c Q N(c(\alpha t-\beta))<0 .
\end{aligned}
$$

Lemma 3.4 Assume that $\left(H_{1}\right)$ and $\left(H_{2}\right)$ hold. Then the set

$$
\Omega_{1}=\left\{u \in \operatorname{dom} M: M u=N_{\lambda} u, \lambda \in(0,1)\right\}
$$

is bounded.

Proof Since $u \in \Omega_{1}, Q N_{\lambda} u=0$. By $\left(H_{1}\right)$, there exists $t_{0} \in[0,1]$ such that $\left|u\left(t_{0}\right)\right| \leq M_{0}$, $\left|u^{\prime}\left(t_{0}\right)\right| \leq M_{0}$. It follows from

$$
u^{\prime}(t)=\int_{t_{0}}^{t} u^{\prime \prime}(s) d s+u^{\prime}\left(t_{0}\right) \quad \text { and } \quad u(t)=\int_{t_{0}}^{t} u^{\prime}(s) d s+u\left(t_{0}\right)
$$

that

$$
\left|u^{\prime}(t)\right| \leq M_{0}+\left\|u^{\prime \prime}\right\|_{0}, \quad|u(t)| \leq 2 M_{0}+\left\|u^{\prime \prime}\right\|_{0} .
$$

Based on $M u=N_{\lambda} u$ and $\left(H_{2}\right)$, we get

$$
\begin{aligned}
\left|\varphi_{p}\left(u^{\prime \prime}\right)\right| & =\left|\lambda \int_{0}^{t} N u(s) d s\right| \\
& \leq\|a\|_{1}+\|b\|_{1} \varphi_{p}\left(2 M_{0}+\left\|u^{\prime \prime}\right\|_{0}\right)+\|c\|_{1} \varphi_{p}\left(M_{0}+\left\|u^{\prime \prime}\right\|_{0}\right)+\|d\|_{1} \varphi_{p}\left(\left\|u^{\prime \prime}\right\|_{0}\right) .
\end{aligned}
$$

If $1<p \leq 2$, by (2.1), we have

$$
\left|\varphi_{p}\left(u^{\prime \prime}\right)\right| \leq\|a\|_{1}+\left(2\|b\|_{1}+\|c\|_{1}\right) M_{0}^{p-1}+\left(\|b\|_{1}+\|c\|_{1}+\|d\|_{1}\right) \varphi_{p}\left(\left\|u^{\prime \prime}\right\|_{0}\right) .
$$

Thus,

$$
\left\|u^{\prime \prime}\right\|_{0} \leq \varphi_{q}\left(\frac{\|a\|_{1}+\left(2\|b\|_{1}+\|c\|_{1}\right) M_{0}^{p-1}}{1-\left(\|b\|_{1}+\|c\|_{1}+\|d\|_{1}\right)}\right) .
$$

Similarly, if $p>2$, then

$$
\left\|u^{\prime \prime}\right\|_{0} \leq \varphi_{q}\left(\frac{\|a\|_{1}+\left(2^{p-1}\|b\|_{1}+\|c\|_{1}\right) 2^{p-2} M_{0}^{p-1}}{\left.1-2^{p-2}\left(\|b\|_{1}+\|c\|_{1}\right)-\|d\|_{1}\right)}\right) .
$$

The above inequalities, together with (3.4), imply that $\Omega_{1}$ is bounded.

Lemma 3.5 Assume that $\left(H_{3}\right)$ holds. Then the set

$$
\Omega_{2}=\{u \in \operatorname{Ker} M: Q N u=0\}
$$

is bounded. 
Proof If $u \in \Omega_{2}$, then $u_{c}(t)=c(\alpha t-\beta)$ and $F\left(N u_{c}\right)=0$. By $\left(H_{3}\right)$, we get $|c| \leq M_{1}$. This means that $\Omega_{2}$ is bounded.

Theorem 3.6 Assume that $\left(A_{0}\right)-\left(A_{2}\right)$ and $\left(H_{1}\right)-\left(H_{3}\right)$ hold. Then the functional boundary value problem (1.1) has at least one solution.

Proof Choose $R_{0}$ large enough such that $\Omega=\left\{u \in X:\|u\|<R_{0}\right\} \supset \bar{\Omega}_{1} \cup \bar{\Omega}_{2}$ and $R_{0}>$ $M_{1}(|\alpha|+|\beta|)$. By Lemma 3.4, $M u \neq N_{\lambda} u$ for $u \in \partial \Omega \cap \operatorname{Ker} M, \lambda \in(0,1)$. So, $\left(C_{1}\right)$ of Theorem 2.4 holds.

Let $H(u, \delta)=\rho \delta u+(1-\delta) J Q N u, u \in \operatorname{Ker} M \cap \bar{\Omega}, \delta \in[0,1]$, where $J: \operatorname{Im} Q \rightarrow \operatorname{Ker} M$ is a homeomorphism with $J(c)=c(\alpha t-\beta)$, and $\rho=1$ or $\rho=-1$, if (3.2) or (3.3) hold, respectively.

For $u \in \operatorname{Ker} M \cap \partial \Omega, u=c(\alpha t-\beta) \neq 0, H(u, 1)=\rho c(\alpha t-\beta) \neq 0$. By Lemma 3.5, we know that $H(u, 0)=Q N(c(\alpha t-\beta))(\alpha t-\beta) \neq 0$. For $\delta \in(0,1), u=c(\alpha t-\beta) \in \operatorname{Ker} M \cap \partial \Omega,\|u\|=$ $R_{0} \leq|c|(|\alpha|+|\beta|)$, we have $|c|>M_{1}$. If $H(c(\alpha t-\beta), \delta)=\rho \delta c(\alpha t-\beta)+(1-\delta) Q N(c(\alpha t-$ $\beta))(\alpha t-\beta)=0$, by $\left(H_{3}\right)$, we obtain

$$
c^{2}=-\frac{1-\delta}{\delta} \rho c \cdot Q N(c(\alpha t-\beta))<0,
$$

which is a contradiction. Thus, $H(u, \delta) \neq 0, u \in \operatorname{Ker} M \cap \partial \Omega, \delta \in[0,1]$.

By invariance of degree under a homotopy,

$$
\begin{aligned}
\operatorname{deg}(J Q N, \Omega \cap \operatorname{Ker} M, 0) & =\operatorname{deg}(H(\cdot, 0), \Omega \cap \operatorname{Ker} M, 0) \\
& =\operatorname{deg}(H(\cdot, 1), \Omega \cap \operatorname{Ker} M, 0) \\
& =\operatorname{deg}(\rho I, \Omega \cap \operatorname{Ker} M, 0)= \pm 1 \neq 0 .
\end{aligned}
$$

By Theorem 2.4, the problem (1.1) has at least one solution in $\bar{\Omega}$.

In the next results the inequality $|u(t)|+u^{\prime}(t) \mid>M$ of $\left(H_{1}\right)$ is replaced with either $|u(t)|>$ $M$ or $\left|u^{\prime}(t)\right|>M$, which will lead to slight modifications of the proof of Lemma 3.4. We recall that $\alpha^{2}+\beta^{2} \neq 0$.

Lemma 3.7 Assume that $\alpha \neq 0$ and the following conditions hold:

$\left(H_{4}\right)$ There exists a constant $M_{2}>0$ such that if $\left|u^{\prime}(t)\right|>M_{2}$, then $F(N u) \neq 0$.

$\left(H_{5}\right)$ There exist functions $a, b, c, d \in C[0,1]$ such that

$$
|f(t, u, v, w)| \leq a(t)+b(t) \varphi_{p}(|u|)+c(t) \varphi_{p}(|v|)+d(t) \varphi_{p}(|w|), \quad t \in[0,1], u, v, w \in \mathbb{R}
$$

and

$$
\begin{aligned}
(2 & \left.+\frac{|\beta|}{|\alpha|}\right)\left(1+\frac{k_{1}(|\alpha|+|\beta|)}{\alpha^{2}+\beta^{2}}\right)\left(\|b\|_{0}+\|c\|_{0}+\|d\|_{0}\right)^{q-1} \\
& < \begin{cases}2^{3-2 q}, & \text { if } 1<p \leq 2, \\
2^{1-q}, & \text { if } p>2 .\end{cases}
\end{aligned}
$$


Then the set

$$
\Omega_{1}=\left\{u \in \operatorname{dom} M: M u=N_{\lambda} u, \lambda \in(0,1)\right\}
$$

is bounded.

Proof For $u \in \Omega_{1}, Q N u=0$. Following the proof of Lemma 3.3 and applying $\left(H_{4}\right)$, we obtain $R(u, \lambda)=(I-P) u$ and a constant $t_{2} \in[0,1]$ such that $\left|u^{\prime}\left(t_{2}\right)\right| \leq M_{2}$.

Since $u(t)=P u(t)+(I-P) u(t)=P u(t)+R(u, \lambda),\left|(P u)^{\prime}\left(t_{2}\right)\right| \leq M_{2}+\|R(u, \lambda)\|_{X}$. By the definition of $P$, we have

$$
\left|\frac{\alpha u^{\prime}(0)-\beta u(0)}{\alpha^{2}+\beta^{2}}\right| \leq \frac{1}{|\alpha|}\left(M_{2}+\|R(u, \lambda)\|_{X}\right) .
$$

Thus,

$$
\|u\|_{X} \leq\|P u\|_{X}+\|R(u, \lambda)\|_{X} \leq\left(1+\frac{|\beta|}{|\alpha|}\right) M_{2}+\left(2+\frac{|\beta|}{|\alpha|}\right)\|R(u, \lambda)\|_{X} .
$$

Since

$$
\begin{aligned}
\|R(u, \lambda)\|_{X} & \leq\left(1+\frac{k_{1}(|\alpha|+|\beta|)}{\alpha^{2}+\beta^{2}}\right)\left\|\int_{0}^{t}(t-s) \varphi_{q}\left(\int_{0}^{s}(I-Q) N_{\lambda} u(r) d r\right) d s\right\|_{X} \\
& \leq\left(1+\frac{k_{1}(|\alpha|+|\beta|)}{\alpha^{2}+\beta^{2}}\right) 2^{q-1} \varphi_{q}\left(\left\|N_{\lambda} u\right\|_{Y}\right),
\end{aligned}
$$

by $\left(H_{5}\right)$, we have

$$
\begin{aligned}
\|u\|_{X} \leq & \left(1+\frac{|\beta|}{|\alpha|}\right) M_{2} \\
& +2^{q-1}\left(2+\frac{|\beta|}{|\alpha|}\right)\left(1+\frac{k_{1}(|\alpha|+|\beta|)}{\alpha^{2}+\beta^{2}}\right) \\
& \times \varphi_{q}\left(\|a\|_{0}+\left(\|b\|_{0}+\|c\|_{0}+\|d\|_{0}\right) \varphi_{p}\left(\|u\|_{X}\right)\right) .
\end{aligned}
$$

By $\left(H_{5}\right), \Omega_{1}$ is bounded, if $p>2$. With a different constant, the same inequality shows that $\Omega_{1}$ is bounded, if $1<p \leq 2$.

Example Consider

$$
\left(\phi_{p}\left(u^{\prime \prime}(t)\right)\right)^{\prime}=f\left(t, u(t), u^{\prime}(t), u^{\prime \prime}(t)\right), \quad t \in(0,1)
$$

where $p=3 / 2$ and

$$
f\left(t, u(t), u^{\prime}(t), u^{\prime \prime}(t)\right)=t+A \sin (\sqrt{|u(t)|})+A \frac{u^{\prime}(t)+1}{\left|u^{\prime}(t)\right|+1} \sqrt{\left|u^{\prime}(t)\right|}+A \sin \left(\sqrt{\left|u^{\prime \prime}(t)\right|}\right)
$$

where $A=0.043$. 
We impose the functional conditions

$$
u^{\prime \prime}(0)=0, \quad B_{1}(u)=u^{\prime}(0)+2 \int_{0}^{1} u(s) d s=0, \quad B_{2}(u)=u(1)=0 .
$$

Then the functional problem is at resonance with $B_{1}(1)=B_{1}(t)=2, B_{2}(1)=B_{2}(t)=1, k=$ $1 / 2, k_{1}=3, \operatorname{Ker} M=\{c(t-1): c \in \mathbb{R}\}$. In this case, $\alpha=\beta=2$ and $\|b\|_{0}=\|c\|_{0}=\|d\|_{0}=A$ and $q=3$. Moreover,

$$
2^{2 q-3}\left(2+\frac{|\beta|}{|\alpha|}\right)\left(1+\frac{k_{1}(|\alpha|+|\beta|)}{\alpha^{2}+\beta^{2}}\right)\left(\|b\|_{0}+\|c\|_{0}+\|d\|_{0}\right)^{q-1}=540 A^{2}<1 .
$$

Clearly,

$$
\begin{aligned}
|f(t, u, v, w)| & \leq t+A \sqrt{|u|}+A \sqrt{|v|}+A \sqrt{|w|} \\
& =t+A \phi_{p}(|u|)+A \phi_{p}(|v|)+A \phi_{p}(|w|), \quad t \in(0,1) .
\end{aligned}
$$

For convenience, introduce

$$
Y(s)=\phi_{q}\left(\int_{0}^{s} f\left(r, u(r), u^{\prime}(r), u^{\prime \prime}(r)\right) d r\right) .
$$

Hence

$$
\begin{aligned}
F(N u) & =\left(B_{2}-k B_{1}\right)\left(\int_{0}^{t}(t-s) Y(s) d s\right) \\
& =\int_{0}^{1}(1-s) Y(s) d s-\int_{0}^{1}\left(\int_{0}^{s}(s-r) Y(r) d r\right) d s \\
& =\int_{0}^{1}(1-s) Y(s) d s-\frac{1}{2} \int_{0}^{1}(1-s)^{2} Y(s) d s \\
& =\frac{1}{2} \int_{0}^{1}\left(1-s^{2}\right) Y(s) d s .
\end{aligned}
$$

If $u^{\prime}(t)>M_{0}>\left(2+\frac{1}{A}\right)^{2}$, then

$$
\frac{u^{\prime}(t)+1}{\left|u^{\prime}(t)\right|+1} \sqrt{\left|u^{\prime}(t)\right|}>\sqrt{M_{0}}
$$

and

$$
f\left(t, u(t), u^{\prime}(t), u^{\prime \prime}(t)\right)>-2 A+A \sqrt{M_{0}}>0 .
$$

If $u^{\prime}(t)<-M_{0}$, then

$$
\frac{u^{\prime}(t)+1}{\left|u^{\prime}(t)\right|+1} \sqrt{\left|u^{\prime}(t)\right|}<-\sqrt{M_{0}}
$$

and

$$
f\left(t, u(t), u^{\prime}(t), u^{\prime \prime}(t)\right)<1+2 A-A \sqrt{M_{0}}<0 .
$$


Hence, $\left|u^{\prime}(t)\right|>M_{0}$ guarantees $|Y(s)|>0$, which, in turn, implies that $F(N u) \neq 0$. Similarly, one can choose $M_{1}>0$ such that, for $u_{c}(t)=c(t-1)$,

$$
F\left(N u_{c}\right)=\left(B_{2}-k B_{1}\right)\left(\int_{0}^{t}(t-s) \phi_{q}\left(\int_{0}^{s} f(r, c(r-1), c, 0) d r\right) d s\right) \neq 0
$$

provided $|c|>M_{1}$.

The above computations show that there is a solution whose existence is governed by Lemma 3.7 .

Lemma 3.8 Assume that $\alpha=0$ and the following conditions hold:

$\left(H_{6}\right)$ There exists a constant $M_{3}>0$ such that if $|u(t)|>M_{3}$, then $F(N u) \neq 0$.

$\left(H_{7}\right)$ There exist functions $a, b, c, d \in C[0,1]$ such that

$$
|f(t, u, v, w)| \leq a(t)+b(t) \varphi_{p}(|u|)+c(t) \varphi_{p}(|v|)+d(t) \varphi_{p}(|w|), \quad t \in[0,1], u, v, w \in \mathbb{R},
$$

and

$$
\left(1+\frac{k_{1}}{|\beta|}\right)\left(\|b\|_{0}+\|c\|_{0}+\|d\|_{0}\right)^{q-1}< \begin{cases}4^{1-q}, & \text { if } 1<p \leq 2, \\ 2^{-q}, & \text { if } p>2 .\end{cases}
$$

Then the set

$$
\Omega_{1}=\left\{u \in \operatorname{dom} M: M u=N_{\lambda} u, \lambda \in(0,1)\right\}
$$

is bounded.

Proof As in the proof of Lemma 3.3, by $\left(H_{6}\right)$, we have $R(u, \lambda)=(I-P) u$ and a constant $t_{3} \in$ $[0,1]$ such that $\left|u\left(t_{3}\right)\right| \leq M_{3}$. Since $u(t)=P u(t)+(I-P) u(t)=P u(t)+R(u, \lambda),\left|(P u)\left(t_{3}\right)\right| \leq$ $M_{3}+\|R(u, \lambda)\|_{X}$ and

$$
\|u\|_{X} \leq\|P u\|_{X}+\|(I-P) u\|_{X} \leq M_{3}+2\|R(u, \lambda)\|_{X} .
$$

Since

$$
\begin{aligned}
\|R(u, \lambda)\|_{X} & \leq\left(1+\frac{k_{1}}{|\beta|}\right)\left\|\int_{0}^{t}(t-s) \varphi_{q}\left(\int_{0}^{s}(I-Q) N_{\lambda} u(r) d r\right) d s\right\|_{X} \\
& \leq\left(1+\frac{k_{1}}{|\beta|}\right) 2^{q-1} \varphi_{q}\left(\left\|N_{\lambda} u\right\|_{Y}\right),
\end{aligned}
$$

by $\left(H_{7}\right)$, we have

$$
\|u\| \leq M_{3}+2^{q}\left(1+\frac{k_{1}}{|\beta|}\right) \varphi_{q}\left(\|a\|_{0}+\left(\|b\|_{0}+\|c\|_{0}+\|d\|_{0}\right) \varphi_{p}\left(\|u\|_{X}\right)\right) .
$$

This, together with $\left(H_{7}\right)$, means that $\Omega_{1}$ is bounded in the case $p>2$ and, similarly, for $1<p \leq 2$.

The proofs of the following theorems are similar to that of Theorem 3.6. 
Theorem 3.9 Assume that $\alpha \neq 0,\left(A_{0}\right)-\left(A_{2}\right)$ and $\left(H_{3}\right)-\left(H_{5}\right)$ hold. Then the functional boundary value problem (1.1) has at least one solution.

Theorem 3.10 Assume that $\alpha=0,\left(A_{0}\right)-\left(A_{2}\right)$ and $\left(H_{3}\right),\left(H_{6}\right),\left(H_{7}\right)$ hold. Then the functional boundary value problem (1.1) has at least one solution.

\section{Conclusion}

We obtain the existence of solution for a third-order functional $p$-Laplacian boundary value problem at resonance. This result extends many existent results and generalizes many related problems in the literature.

\section{Acknowledgements}

The author would like to appreciate the anonymous reviewer for careful reading and very useful comments.

Funding

This work is supported by the Natural Science Foundation of China (11775169) and the Natural Science Foundation of Hebei Province (A2018208171)

\section{Availability of data and materials}

Not applicable.

\section{Competing interests}

The authors declare that none of them have any competing interests in the manuscript.

Authors' contributions

The authors declare that they carried out all the work in this manuscript and read and approved the final manuscript.

\section{Author details}

${ }^{1}$ College of Sciences, Hebei University of Science and Technology, Shijiazhuang, P.R. China. ${ }^{2}$ Department of Mathematics and Statistics, University of Arkansas at Little Rock, Little Rock, USA.

\section{Publisher's Note}

Springer Nature remains neutral with regard to jurisdictional claims in published maps and institutional affiliations.

Received: 20 January 2018 Accepted: 25 April 2018 Published online: 08 May 2018

\section{References}

1. Mawhin, J.: Topological Degree Methods in Nonlinear Boundary Value Problems. NSF-CBMS Regional Conference Series in Math., vol. 40. Am. Math. Soc., Providence (1979)

2. Ge, W., Ren, J: An extension of Mawhin's continuation theorem and its application to boundary value problems with a p-Laplacian. Nonlinear Anal. 58, 477-488 (2004)

3. Du, Z., Lin, X., Ge, W.: Some higher-order multi-point boundary value problem at resonance. J. Comput. Appl. Math. $177,55-65$ (2005)

4. Jiang, W.: Solvability of fractional differential equations with $p$-Laplacian at resonance. Appl. Math. Comput. 260, 48-56 (2015)

5. Jiang, W.: Solvability for $p$-Laplacian boundary value problem at resonance on the half-line. Bound. Value Probl. 2013 207 (2013). https://doi.org/10.1186/1687-2770-2013-207

6. Kosmatov, N., Jiang, W.: Second-order functional problems with a resonance of dimension one. Differ. Equ. Appl. 8(3), 349-365 (2016)

7. Phung, P.D., Truong, L.X.: On the existence of a three point boundary value problem at resonance in $\mathbb{R}^{n}$. J. Math. Anal. Appl. 416, 522-533 (2014)

8. Zhang, X., Feng, M., Ge, W.: Existence result of second-order differential equations with integral boundary conditions at resonance. J. Math. Anal. Appl. 353, 311-319 (2009)

9. Zhao, Z., Liang, J.: Existence of solutions to functional boundary value problem of second-order nonlinear differential equation. J. Math. Anal. Appl. 373, 614-634 (2011)

10. Zou, Y., Cui, Y.: Existence results for a functional boundary value problem of fractional differential equations. Adv. Differ. Equ. 2013, 233 (2013)

11. Han, W., Kang, S.: Multiple positive solutions of nonlinear third-order BVP for a class of $p$-Laplacian dynamic equations on time scales. Math. Comput. Model. 49, 527-535 (2009)

12. Royden, H.L.: Real Analysis, 3rd edn. Prentice Hall, Englewood Cliffs (1988) 